\title{
ESTUDIO DE LA APLICACIÓN DE LA PLATAFORMA DE INTRANET EN LA GESTIÓN DEL CONOCIMIENTO DE LA INSTITUCIÓN EDUCATIVA “JOSÉ
} ABELARDO QUIÑONES” DE ATE, LIMA-PERÚ

Study of the implementation application of the platform of intranet in the management of the knowledge of the educational institution "José Abelardo Quiñones" de Ate, Lima-Peru

\author{
Isaac Huaira Paitán ${ }^{1}$ \\ Institución Educativa Pública No. 1138
}

Recibido: 24-2-2014

Aceptado: 25-6-2014

\begin{abstract}
RESUMEN
Los docentes de educación secundaria son básicamente transmisores de información y conocimientos, al no explotar adecuadamente la intranet como una herramienta de la gestión del conocimiento para desarrollar el conocimiento tácito y explicito. En este contexto, la hipótesis planteada fue: ¿Existe relación entre el sistema de intranet con la gestión del conocimiento en los docentes y alumnos de educación secundaria de la Institución Educativa “JOSÉ ABELARDO QUINOONES" Urb. Valdivieso-Ate? La investigación fue no experimental de diseño correlacional, transversal al aplicarse en un sólo momento los cuestionarios a los alumnos y docentes, los mismos que obtuvieron una confiabilidad de $\alpha=0.909$ y $\alpha=0.946$, respectivamente y una validez de contenido de $90 \%$. La muestra en los alumnos $(n=180)$ fue probabilística y estratificada y, en los docentes $(n=30)$ no probabilista y censal. Para el análisis y contrastación de la hipótesis general y específicas, se realizó la prueba de significatividad y, con el coeficiente de correlación de Spearman se determinó la correlación altamente significativa entre el sistema intranet y gestión del conocimiento $(\rho=0,872)$, así como la creación del conocimiento $(\rho=0,801)$, con el almacenamiento y recuperación del conocimiento $(\rho=0,864)$, intercambio de conocimiento $(\rho=0,859)$ y uso del conocimiento $(\rho=0,905)$. Al respecto, López (2011) refiere que los profesores valoran el desarrollo de las habilidades personales de los alumnos a través del trabajo colaborativo con la Wikipedia. Para Hopkins (2006), los profesores al utilizar estrategias de gestión de conocimiento mejoran sus métodos de enseñanza, redundando en la mejora del proceso de aprendizaje de los estudiantes.
\end{abstract}

Palabras Clave: Intranet, gestión del conocimiento, institución educativa.

\section{Abstract}

Teachers of secondary education are basically transmitters of information and knowledge, not to properly exploit the intranet as a knowledge management tool to develop tacit knowledge and explicit. In this context, the hypothesis was: There is a relationship between the system of intranet knowledge management on teachers and students in secondary education from the Educational Institution "JOSÉ ABELARDO QUIÑONES" AV. Valdivieso-Ate. Research is non-experimental correlation, cross design applies in an only time questionnaires to students and teachers, those who obtained a reliability of $\alpha=0.909$ and $\alpha=0.946$, respectively, and validity of content of $90 \%$. Students in the sample $(\mathrm{n}=180)$ was probabilistic and stratified, and teachers $(\mathrm{n}=30)$ non-probabilistic

1 Magister en Administración de la Educación, docente del nivel secundaria en el Área de Matemática en la Institución Educativa No 1138, Urb. Valdiviezo distrito de Ate-Lima. E-mail: isaachuaira@gmail.com 
and census. Analysis and verification of general and specific hypothesis, was performed significance test and Spearman's rank correlation coefficient is determined highly significant correlation between intranet and knowledge management $(\rho=0,872)$ as well as the creation of knowledge $(\rho=0,801)$ with the storage and retrieval of knowledge $(\rho=0,864)$, exchange of knowledge $(\rho=0,859)$, use of knowledge $(\rho=0,905)$. In this regard, López (2011) concerns that teacher's value the development of personal skills of the students through collaborative work with the Wiki. For Hopkins (2006), teachers using strategies of knowledge management improve their teaching methods, resulting in the improvement of the learning process of the students.

Keywords: TIC, public administration, society of the information, public management.

\section{INTRODUCCIÓN}

La gestión del conocimiento constituye una línea de pensamiento para entender las formas de creación de ventajas competitivas en el complejo mundo globalizado, la cual analiza como los activos de conocimiento en cualquier organización y su utilización puede elevar la capacidad de competir. Proceso que actualmente en el interior de las instituciones, como las educativas, es facilitado por la intranet que es un sistema que permite producir, almacenar y recuperar información y conocimientos. Desde esta perspectiva los alumnos de educación secundaria con el sistema de intranet acceden a la información, documentos, manuales teóricos que se generan en su institución y que es necesario establecer su relación con la gestión del conocimiento a fin que tengan conocimientos en el contexto del aprendizaje significativo. Pero, se tiene que los docentes de educación secundaria básicamente son transmisores de información y conocimientos y no explotan adecuadamente el sistema de cómputo para desarrollar el conocimiento tácito y explicito a través de la intranet como una herramienta de la gestión del conocimiento. Por lo que en la presente investigación fue determinar la relación entre el sistema de intranet con la gestión del conocimiento en los docentes y alumnos de educación secundaria de la Institución Educativa "José Abelardo Quiñones”. Y de manera específica el establecer la relación entre el sistema intranet con la creación del conocimiento en la gestión del conocimiento en los docentes y alumnos de educación secundaria, la relación entre el sistema de intranet con el almacenamiento y recuperación del conocimiento en la gestión del conocimiento en los docentes y alumnos de educación secundaria, al igual que determinar la relación entre el sistema de intranet con el intercambio de conocimiento en la gestión del conocimiento en los docentes y alumnos de educación secundaria y como último objetivo el establecer la relación entre el sistema de intranet con el uso del conocimiento en la gestión del conocimiento en los docentes y alumnos de educación secundaria. Derivándose como hipótesis general: Existe relación entre el sistema de intranet con la gestión del conocimiento en los docentes y alumnos de educación secundaria de la Institución Educativa “José Abelardo Quiñones” Urb. Valdiviezo-Ate-Perú.

\section{ANTECEDENTES}

Entre los antecedentes de la investigación se tiene a Ruiz (2006), encontró que en los colegios de la ciudad de Piura existen problemas en el manejo y procesamiento de información, problemas de comunicación con los padres de familia para informarles del rendimiento académico de sus hijos. El 55,6\% de los docentes utilizan el software para calcular el promedio de un curso para un periodo académico; en los colegios el $44,4 \%$ de las computadoras dispone de internet, el $66,7 \%$ cuenta con un software de apoyo a la gestión académica. Concluyendo que la aplicación del intranet fue planteada como una aplicación web para permitir el acceso a ésta desde cualquier punto de internet.

O'Brien y Marakas (2008), señalan que las tecnologías y redes comunicacionales, como Internet, intranets y extranets en sus inicios tuvo aplicaciones educativas. Aspecto que es tomado por Joo (2004), quien refiere que la tecnología de la información y de la comunicación (TIC), entendida como el Internet, Intranet, software y com- 
putadoras, se inició en el plano educativo. Señala que el 18,18\% domina el tema de la gestión del aprendizaje con las TIC. Y que a nivel de docentes, si no se exige el uso de la Intranet como medio para organizar y generar espacios para compartir, ésta se convierte en una especie de "gran disco duro" donde cada uno ve la mejor manera de "organizar sus cosas", usando la Intranet para no perder información en el archivo C de sus máquinas; pero, contrariamente, los administrativos no usan la red, sino su carpeta en el disco duro para no perder tiempo y tener más rápido el acceso a la información, restándole el potencial para compartir, crear y recrear.

Donate y Guadamillas (2010) desde el enfoque del conocimiento analizan la manera en que las empresas formulan e implantan sus Estrategias de Gestión del Conocimiento (EGC), al explorar los patrones específicos de EGC encontraron que existen diferencias entre las empresas tanto en el diseño e implantación de la EGC como en la actitud innovadora desarrollada, donde la orientación tecnológica obtiene 0,872 del estadístico de Levene, en el desarrollo interno de tecnología $(2,767)$, en la incorporación de tecnología externa $(0,628)$.

\section{INTRANET}

O’Brien y Marakas (2008) refieren que una Intranet es una red dentro de una organización que utiliza tecnologías de internet (tales como navegadores y servidores web, protocolos TCP/IP de red, base de datos y edición de documentos HTML de hipermedios, entre otros) para proporcionar un ambiente parecido a Internet dentro de la organización para compartir información, comunicarse, colaborar y apoyar los procesos de la organización. Para Abad (2010) Intranet es una red perteneciente sólo a una organización, diseñada y desarrollada en base a los protocolos característicos de Internet -como el protocolo TCP/IP-, donde una computadora al actuar como servidor permite que un conjunto de computadoras personales estén conectadas entre sí y que en tiempo real internamente tengan acceso al mismo tipo de contenidos que la organización pone a disposición de sus usuarios.
Por su parte Pérez y Solana (2009) señalan dos tendencias básicas: la visión técnica, entre la que se puede mencionar la definición ofrecida por O’Brien y Marakas (2008) y el segundo enfoque, que es el funcional, y bajo esta percepción se tiene que intranet es una "organización de aprendizaje, que posibilita la integración de gente, procesos, procedimientos y principios para formar una cultura intelectualmente creativa que permita la implantación de la efectividad total de la organización" (Cascante y Fonseca , 2008), estos mismos autores refieren que la Intranet es una red interna de la organización donde, basada en la misma plataforma tecnológica de Internet, sus usuarios comparten recursos, información y servicios, facilitando la comunicación, colaboración y coordinación utilitaria interna. Con la intranet se facilita la eficiencia de los procesos de la organización, ello sin restricción del tamaño y ubicación de la organización. La funcionalidad de Intranet puede darse de manera aislada o no a Internet. Cabanas citado por Carrillo, Castillo y Gómez (2010), señala que Intranet es un sistema de comunicación interactivo de la organización conformado por un conjunto de procesadores conectados mediante un servidor posibilita compartir información. Apórtela (2007), puntualiza que una Intranet trasciende el sistema técnico, porque la gestión, los involucrados en las actividades de la organización, es enmarcada con otra filosofía, como que la institución sea más competitiva y se adecue a los requerimientos de los clientes.

En una institución educativa, Intranet ofrece (Abad 2010):

1. La capacidad de compartir recursos -impresoras, escáner, entre otros-, así como tener mayor comunicación a través de Internet.

2. Que la institución educativa, estudiantes y docentes estén en páginas web que dinamice la consulta entre ellos.

3. Que los usuarios tengan espacios en el disco virtual que les permita guardar y recuperar información desde cualquier punto de la red interna y externa.

4. Servicio de correo electrónico, servicio de mensajería instantánea, entre otras.

5. Los usuarios de la institución educativa a través de foros podrán intercambiar experiencias, 
opiniones.

6. Los usuarios autorizados a través de diversos instrumentos gestionan tutorías, elaboran de plantillas educativas, test, muros virtuales; gestión administrativa de la institución, como la solicitud de certificados, el proceso de la matricula, publicación de los rendimientos escolares, entre otros aspectos.

Con el Intranet se puede crear y editar publicaciones digitales e impresas de sus usuarios y ponerlas al alcance de la comunidad educativa, así como organizar y publicar información referente a la institución educativa y de sus miembros para utilizarla oportunamente y para la toma de decisiones.

En suma, Intranet es una red de información interna, de acceso restringido a los usuarios de la organización que la tiene, dentro de los parámetros de seguridad; donde fluye la comunicación, la información, la colaboración y coordinación que posibiliten mejorar la integración de los procedimientos, procesos y miembros de una institución educativa.

\section{GESTIÓN DE CONOCIMIENTO}

El ser humano, refiere Méndez (2011), se caracteriza por su permanente actividad de conocer -que es un proceso intelectual por el cual se establece una relación entre quien conoce y el objeto conocido-, sea por su dotación física, el cerebro o por su innata perspicacia por saber más de sí mismo y de todo su micro y macromundo. Sabino (2007) señala que el hombre es un ser preocupado constantemente por conocer el mundo que lo rodea, sus leyes, su sentido y devenir. Desde que el ser humano tiene uso de razón, la reflexión por conocer lo que sucede a su alrededor ha sido y es una de sus principales preocupaciones. Nonaka y Takeuchi (2008) sostienen que el conocimiento es de naturaleza subjetiva e intuitiva, con una dimensión cognoscitiva (que incluye los esquemas, modelos mentales, creencias y percepciones arraigadas en cada persona), y que refleja la imagen de la realidad (lo que existe, lo que es) y nuestra visión de futuro (lo que debería ser), de manera que controlan la forma en que se percibe el mundo que nos rodea. Las ideas personales, la intui- ción, elementos por demás subjetivos, son parte integral del conocimiento, el cual incluye valores, ideales y emociones, así como símbolos e imágenes. Estos autores introducen el conocimiento organizacional, sosteniendo que el conocimiento es principalmente "tácito" ("algo muy evidente y difícil de expresa"), este tipo de conocimiento es muy personal y es difícil de plantear a través del lenguaje formal, no es fácil transmitirlo y compartirlo con otros, debido a que está compuesta por las corazonadas, subjetividades, intuición y las ideas.

De allí, que el conocimiento es el conjunto de experiencias, saberes, valores, información, percepciones e ideas que crean determinada estructura mental en el sujeto para evaluar e incorporar nuevas ideas, saberes y experiencias. Y cuando el conocimiento es tomado como la dirección planificada y continua de procesos y actividades para potenciar el conocimiento e incrementar la competitividad a través del mejor uso y creación de recursos del conocimiento individual y colectivo se está hablando de la gestión del conocimiento.

Para que una organización logre ampliar su competitividad, satisfaga a sus clientes y compita adecuadamente, la gestión del conocimiento es un factor crítico de éxito, ello porque con los sistemas de información a través de la Intranet se maneja, trata y usa el conocimiento en las instituciones facilita el acceso de la información y del conocimiento para dinamizar los procesos organizacionales y de esta manera el liderazgo de quienes toman las decisiones se fortalezca y destaque.

Soy (2009) aborda la Intranet desde la perspectiva informativa, donde la integración de las rutinas de trabajo que diariamente se dan, se orientan a integrar la información, las políticas y estrategias en fundamentos de la gestión del conocimiento institucional, a fin que la publicación y consumo de la información trascienda hacia una cultura de la información y se valore la función del conocimiento en las organizaciones. Siendo el factor fundamental, el generar valor en la organización a través de la gestión del conocimiento, al proponer dirigir y controlar el proceso productivo. Es esencial la atención y el estudio de los procesos de transferencia de conocimientos vinculados al ci- 
clo de vida de la información: las formas de crear, adquirir, distribuir, usar, almacenar y expurgar información o conocimiento.

"En este sentido, existen dos corrientes sobre gestión del conocimiento. La primera se centra en el incremento del conocimiento y en la reutilización de los depositarios del mismo" (Barney, 2012), basada en la provisión de soluciones tecnológicas (Carrillo, Anumba y Kamara, 2010). "En esta corriente, la gestión del conocimiento se refiere al conjunto de métodos en desarrollo, herramientas, técnicas y valores a través de los cuales las organizaciones pueden adquirir, desarrollar, medir, distribuir y proporcionar un retorno de sus activos intelectuales" (Snowden, 2009). Por su parte, Teece (2010), refiere que la segunda corriente de investigación evalúa los procesos, la estructura organizacional y las aplicaciones TIC que permiten a los individuos influir en su creatividad y capacidades para brindar valor comercial y detectar, y luego aprovechar, oportunidades de manera inmediata y eficaz. En esta corriente de investigación, para Schulz y Jobe (2011), "la gestión del conocimiento se define como un proceso de transformación del conocimiento tácito en conocimiento explícito para facilitar los flujos intelectuales organizacionales". Martínez, Peñalver y Salamanca (2009) apuestan porque la gestión del conocimiento en las organizaciones sean dos retos:

1. Que el conocimiento explícito o codificado -sea en libros artículos, guías, manuales, circulares, procedimientos, etcétera- esté a disposición en el momento justo y en el lugar de trabajo de los miembros de la organización.

2. Que el conocimiento tácito -criterio profesional, institución, profesionalidad, etcéterasea codificado y difundido a fin de dar lugar al conocimiento explícito.

Por otro lado, para Henao y Cardona (2010), la gestión del conocimiento en las organizaciones se basa en las herramientas de las tecnologías de información, entre ellas las intranets; pero es necesario complementarlas con estrategias de integración del manejo de la información a fin crear, mapear, recuperar, usar y aprender el conocimiento, de este modo la introducción de la gestión del conocimiento es facilitada en la organización y la información se constituye en una fuente de creación del conocimiento. Por su parte, Nonaka y Takeuchi (2008) afirman que la gestión del conocimiento organizativo se define como la capacidad de la empresa para crear conocimiento nuevo, diseminarlo en la organización e incorporarlo en productos, servicios y sistemas. Tarí y García (2009) mencionan siete dimensiones de la gestión del conocimiento: La primera referida a la creación de conocimiento (que es el aprendizaje organizativo): que consiste en la adquisición, diseminación e interpretación de la información. La segunda es la transferencia y almacenamiento de conocimiento (que es el conocimiento organizativo): es la parte de la gestión que consiste en almacenar y transferir el conocimiento. La tercera es la aplicación y uso del conocimiento (que es la organización de aprendizaje): está dado por el trabajo en equipo, el promover el diálogo, así como capturar y compartir el aprendizaje. En el interior de una organización, la gestión del conocimiento es considerada como un proceso, el cual está integrado por la generación, codificación, transferencia y utilización del conocimiento (Wiig, 2007).

La cuarta es la generación del conocimiento: que es el proceso de adquisición del conocimiento externo y su creación en las organizaciones, además los conocimientos de una persona los pone en práctica. (Bueno, 2008).

La quinta dimensión es la codificación, almacenamiento o integración del conocimiento: poner al alcance de todos el conocimiento organizativo, ya sea de forma escrita o localizando a la persona que lo concentra.

La sexta dimensión es la transferencia del conocimiento: A través de los mecanismos formales y/o informales de la comunicación se analizan los espacios en que se dan los intercambio del conocimiento (Bueno, 2009).

La sétima dimensión es la utilización del conocimiento: es la aplicación de los conocimientos adquiridos en las actividades diarias dentro de la organización.

El conocimiento tácito tiene un mayor componente en los procesos de la generación y transfe- 
rencia del conocimiento. En la codificación y en la utilización, el conocimiento tácito es convertido en explícito a fin que los miembros de la organización lo comprendan. Bueno (2009) señala que los aspectos esenciales de la gestión del conocimiento son la creación y la distribución de éste, aunque otros autores como Quintana (2006) las matizan en todas las etapas del proceso de la gestión del conocimiento.

Por otro lado, Hopkins (2006) refiere que es deber de las escuelas y docentes crear un ambiente educativo que desarrolle y fortalezca las habilidades cognitivas de los alumnos, que les permita construir y descubrir un conocimiento relevante y significativo, que los involucre en un proceso de investigación y que les permita trabajar en productos que tengan relevancia mucho más allá de la escuela. A pesar de ello, las escuelas, el trabajo de los profesores y las aulas aún no han sido suficientemente rediseñados para atender apropiadamente ni a estos cambios ni a esta generación. En este sentido a través de esta propuesta se colabora con una estrategia de gestión del conocimiento en encontrar los medios para que los profesores aceleren sus procesos de creación de conocimiento, compartan este conocimiento con sus colegas y se genere una espiral positiva de crecimiento y mejora de sus métodos de enseñanza, así como de crecimiento de conocimiento, redundando al final en la mejora del proceso de aprendizaje de los alumnos.

En suma, la gestión del conocimiento es el conjunto de actividades que crean, utilizan, comparten y desarrollan los conocimientos los individuos que trabajan en una organización.

\section{MATERIAL Y MÉTODOS}

\section{Participantes}

La población del estudio estuvo conformada por 338 alumnos (de 3ro., 4to. y 5to. año) y 30 docentes de nivel secundaria de la I.E. No. 1138, Urb. Valdiviezo-Ate, Lima.

La muestra de los alumnos la constituyeron 180 alumnos y 30 docentes. Para el caso de los alumnos, se aplicó el muestreo estratificado proporcional a fin de tener una muestra representativa de toda la población y para los docentes fue una muestra censal, debido a que la población es pequeña.

\begin{tabular}{|cccccc|}
\hline Grado & \multicolumn{5}{c}{ Sección } \\
\hline 3ro. & 16 & 17 & 17 & 16 & 66 \\
\hline 4to. & 16 & 17 & 16 & 16 & 65 \\
\hline 5to. & 17 & 16 & 16 & - & 49 \\
\hline Total & 49 & 50 & 49 & 32 & 180 \\
\hline
\end{tabular}

Tabla 1. Alumnos: composición estratificada de la muestra

\section{Instrumento}

Para la recolección de datos se empleó la técnica de la encuesta, para la cual se utilizó como instrumento el cuestionario para obtener información de la gestión del conocimiento. La encuesta consta de 40 preguntas, 20 corresponden a la variable Intranet y 20 a la variable Gestión del conocimiento, distribuidas según sus dimensiones.

El instrumento de la investigación fue validado por juicios de cinco expertos, quienes dieron sus puntos de vista, correcciones y aprobación sobre el diseńo, estructura y organización del instrumento, obteniendo un porcentaje del $91 \%$.

Para comprobar la confiabilidad del instrumento, se aplicó a una muestra de 30 estudiantes la encuesta, resultando una fiabilidad, según el alfa de Cronbach, $\alpha=0.909$, lo que significa que el instrumento es altamente confiable. Para el caso del instrumento a los docentes se aplicó en una muestra de 20 docentes; siendo el resultado de $\alpha=0.946$ lo que significa que el instrumento también es altamente confiable.

\section{Tipo y diseño}

Teniendo en cuenta los objetivos e hipótesis formuladas el estudio es una investigación correlacional. Es correlacional por que asocia variables mediante un patrón predecible para la muestra antes señalada que es representativa de la población lo que permite su generalización.

El diseño del estudio fue no experimental, transec- 
Estudio de la aplicación de la plataforma de intranet en la gestión del conocimiento de la institución educativa "José Abelardo Quiñones" de Ate, Lima-Perú

\begin{tabular}{|c|c|c|c|}
\hline & Dimensiones & Indicadores & Items \\
\hline \multirow{4}{*}{ Intranet } & \multirow{4}{*}{$\begin{array}{l}\text { Estructura Intranet (endógena y } \\
\text { exógena) }\end{array}$} & $\begin{array}{l}\text { - Calidad y definición de las necesida- } \\
\text { des funcionales }\end{array}$ & 01-05 \\
\hline & & - Desarrollo de guías del Intranet & $06-10$ \\
\hline & & - Actualización de los procesos & $11-14$ \\
\hline & & • Evaluación sistemática & $15-20$ \\
\hline \multirow{6}{*}{$\begin{array}{l}\text { Gestión del } \\
\text { conocimiento }\end{array}$} & Creación de conocimiento & - Tácito & $01-03$ \\
\hline & \multirow{5}{*}{$\begin{array}{l}\text { Almacenamiento y recuperación } \\
\text { del conocimiento } \\
\text { Intercambio del conocimiento } \\
\text { Uso del conocimiento }\end{array}$} & - Explícito & 04-06 \\
\hline & & $\begin{array}{l}\text { - Organización del conocimiento } \\
\text { (codificación) }\end{array}$ & 07-08 \\
\hline & & - Recuperación del conocimiento & $09-10$ \\
\hline & & - Diálogo creativo & $11-15$ \\
\hline & & - Utilización del conocimiento & $16-20$ \\
\hline
\end{tabular}

Tabla 2. Estructura de los instrumentos de recolección de datos de ambas variables

cional (o transversal). Es no experimental, porque el sistema de intranet existente en la institución educativa del estudio y los resultados obtenidos fue en su contexto natural, para luego analizarlos. Es transeccional porque los datos se recolectaron en un solo momento, esto es, cuando se aplicó la encuesta a los docentes y a los alumnos a fin de describir las variables y analizar su incidencia e interrelación en un momento dado.

\section{Procedimiento}

La obtención de la información se realizó a través de una encuesta aplicada ("Intranet para mejorar la gestión del conocimiento") a 180 alumnos de 3ro., 4to. y 5to. Ańo y otra encuesta a 30 docentes de la institución educativa, aplicada en noviembre del 2013. La data del estudio fue procesada por el programa estadístico SPSS v. 19; los resultados se presentan en tablas de frecuencia y porcentajes con sus respectivos gráficos. Se aplicó el análisis de correspondencia múltiple para variables categóricas con puntajes para las dimensiones del sistema intranet y de la gestión del conocimiento. Con el test de prueba de normalidad de Kolmogorov-Smirnov para una muestra $(n>50)$, se determinó que las variables no tienen distribución normal ni están relacionadas linealmente; por ello, para la prueba de las hipótesis se aplicó el $\rho$ de Spearman $\left(\chi^{2}\right)$, a fin de determinar el nivel de correlación entre sus variables. La aceptación o rechazo de la hipótesis fue mediante la signifi- catividad bilateral, donde se acepta la hipótesis: Sig (bilateral) $>0.05$; se rechaza la hipótesis: Sig (bilateral) $<0.05$.

\section{RESULTADOS}

En la tabla 3 se tiene que $\mathrm{p}=0.418$, es decir que $\mathrm{p}$ $>0.05$, entonces se acepta la hipótesis alterna (rechazándose la hipótesis nula), se acepta que existe relación entre el sistema de Intranet con la gestión del conocimiento (hipótesis general); existiendo una correlación lineal entre sistema Intranet organizacional y gestión del conocimiento muy alta $(0.8<\mathrm{r}<1) 87.2 \%$ directamente proporcional (con signo positivo).

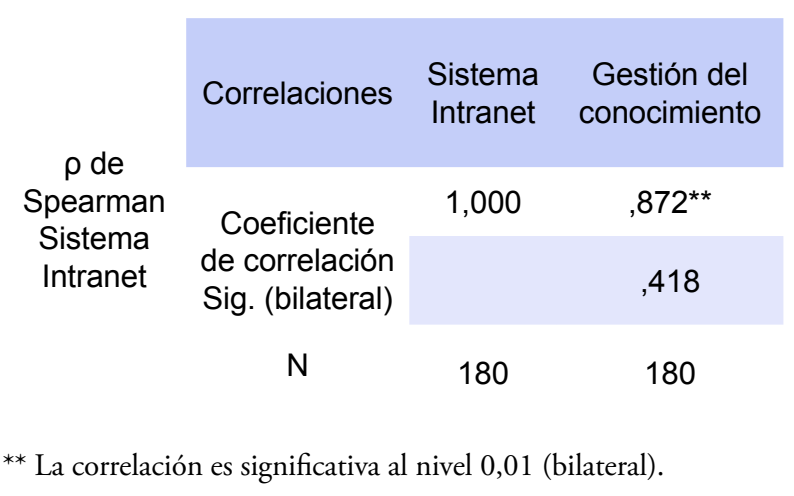

Tabla 3. Correlación entre las variables: sistema Intranet y gestión del conocimiento.

En la tabla 4 se tiene que $\mathrm{p}=0.200$, es decir que $\mathrm{p}>0.05$ entonces se acepta la hipótesis alterna (se 
rechaza la hipótesis nula), se acepta que existe relación entre el sistema de Intranet con el almacenamiento y recuperación del conocimiento en la gestión del conocimiento (hipótesis específica 1); existiendo correlación lineal entre sistema intranet y la creación del conocimiento en la gestión del conocimiento, muy alta $(0.8<\mathrm{r}<1) \quad 80.1 \%$ directamente proporcional (con signo positivo).

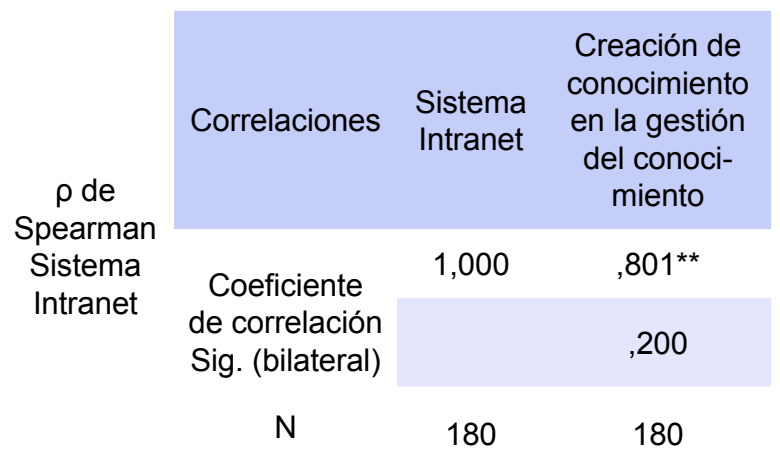

** La correlación es significativa al nivel 0,01 (bilateral).

Tabla 4. Correlación entre las variables: sistema Intranet y almacenamiento y recuperación del conocimiento en la gestión del conocimiento

En la tabla 5 se tiene que $\mathrm{p}=0.384$, es decir que $\mathrm{p}>0.05$, entonces se acepta la hipótesis alterna y que existe relación entre el sistema de intranet con almacenamiento y la recuperación del conocimiento gestión del conocimiento (hipótesis específica 2); existiendo una correlación lineal entre el sistema Intranet y el almacenamiento y recuperación del conocimiento en la gestión del conocimiento, muy alta $(0.8<\mathrm{r}<1) \quad 86.4 \%$ directamente proporcional (con signo positivo).

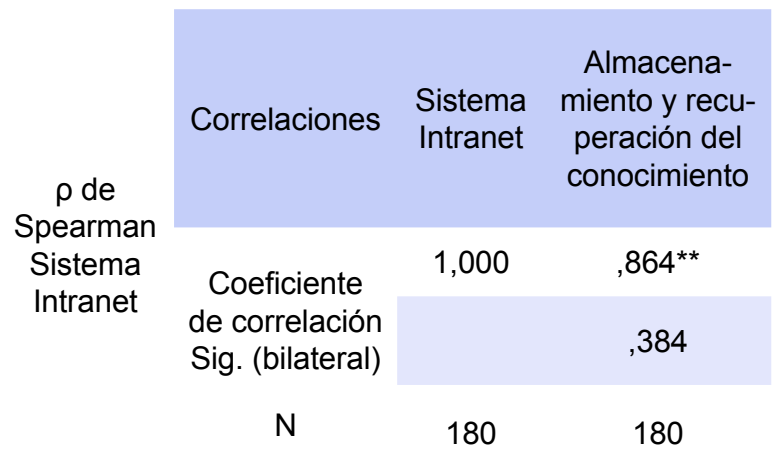

** La correlación es significativa al nivel 0,01 (bilateral).

Tabla 5. Correlación entre las variables: sistema intranet y almacenamiento y recuperación del conocimiento gestión del conocimiento
En la tabla 6 se tiene que $\mathrm{p}=0.493$, es decir, $\mathrm{p}>0.05$, entonces se acepta la hipótesis alterna (rechazándose la hipótesis nula), se acepta que existe relación entre el sistema de Intranet con el intercambio de conocimiento en la gestión del conocimiento (hipótesis específica 3); existiendo correlación lineal entre sistema Intranet y el intercambio de conocimiento, muy alta $(0.8<\mathrm{r}$ $<1) 85.9 \%$ directamente proporcional (con signo positivo).

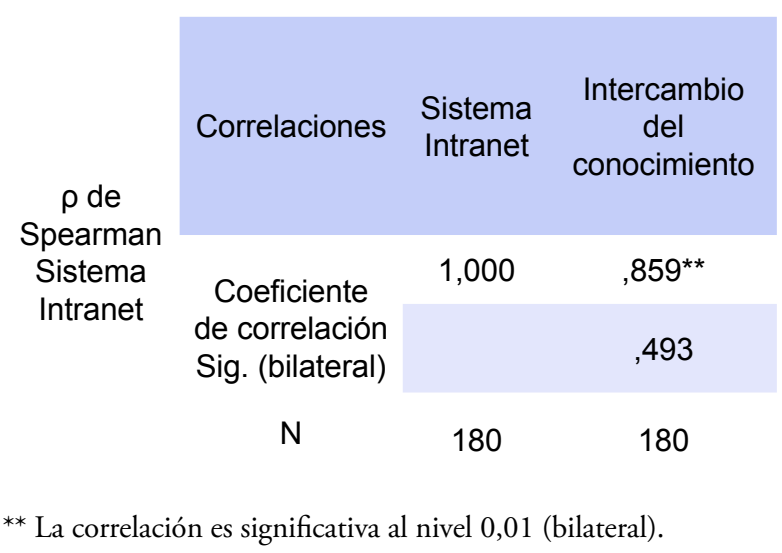

Tabla 6. Correlación entre las variables: sistema Intranet e intercambio de conocimiento

En la tabla 7 se tiene que $\mathrm{p}=0.460$, es decir que $\mathrm{p}>0.05$, entonces se acepta la hipótesis alterna (rechazándose la hipótesis nula), es decir, se acepta que existe relación entre el sistema de intranet con el uso del conocimiento en la gestión del conocimiento (hipótesis específica 4); existiendo una correlación lineal entre sistema Intranet y el uso del conocimiento en la gestión del conocimiento, muy alta $(0.8<\mathrm{r}<1) 90.5 \%$ directamente proporcional (con signo positivo).

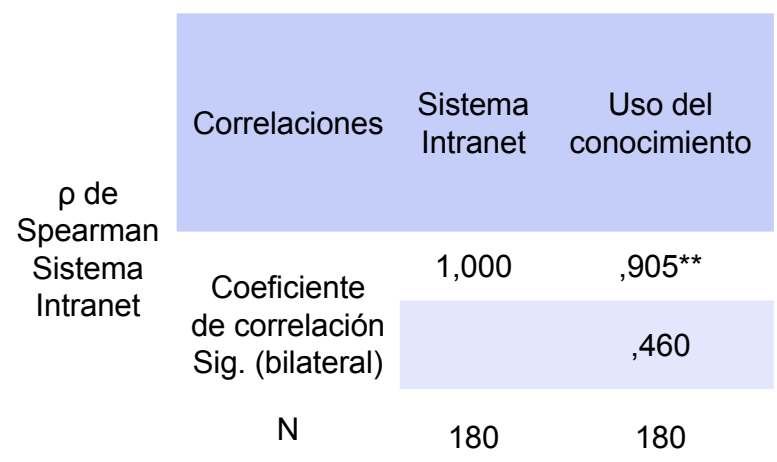

** La correlación es significativa al nivel 0,01 (bilateral).

Tabla 7. Correlación entre las variables: sistema Intranet e uso del conocimiento 


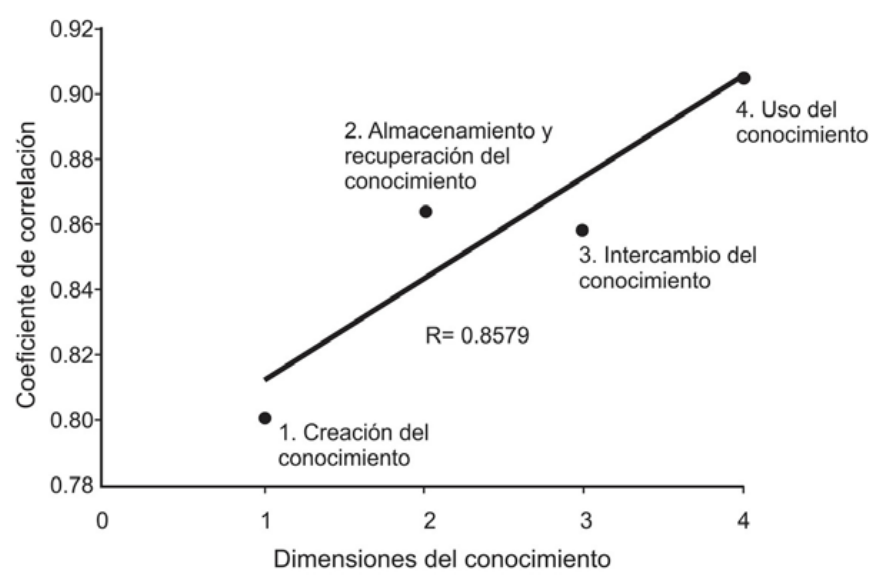

Figura 1. Resultados del coeficiente de correlación entre el sistema de Intranet y las dimensiones del conocimiento. Fuente. Elaborado con el cuestionario aplicado a los docentes y a los alumnos

Con respecto al coeficiente de correlación de Pearson entre el sistema de Intranet y las dimensiones del conocimiento en la gestión del conocimiento (creación del conocimiento, almacenamiento y recuperación del conocimiento, intercambio del conocimiento y el uso del conocimiento) se tiene que el coeficiente de correlación es 0.8579 (Figura 1), es decir que existe una correlación muy alta $(0.8579<\mathrm{r}<1) 85.79 \%$ directamente proporcional (con signo positivo).

\section{DISCUSIÓN Y CONCLUSIONES}

Con la prueba estadística de significatividad bilateral se tiene que las dimensiones del sistema de Intranet están relacionadas con la dimensiones de la gestión del conocimiento, tal es que el intranet de la I.E. le facilita almacenar, recuperar y procesar la información -con una correlación positiva débil 0.303 y un índice de significatividad de 0.105-, asimismo con el Intranet de la Institución Educativa ha mejorado el intercambio de información con sus compañeros, profesores y dirección (Rho de Spearman 0.130 y un índice de significatividad de 0.081), lo cual denota que los alumnos y los docentes hacen uso de la computadora a fin de aprovechar en su beneficio. Esta apreciación no condice con lo encontrado por Joo (2004), quien encontró que existe un $22 \%$ de profesores que no coordinan para hacer uso de los laboratorios y de los software disponibles, evitando el aprovechamiento de las mismas y de mejorar o hacer más ri- cos y motivadores los aprendizajes de los alumnos, sin embargo señala que "la computadora es un medio de expresión personal e instrumento para el propio desarrollo intelectual. En un proceso que involucra personas, máquinas e ideas, interrelacionadas entre ellas, en el trato con la computadora, la creatividad del alumno toma forma en la pantalla, exteriorizada en la libertad manifestada por su voluntad creadora ante el ratón, un programa, ante los conceptos conocidos y por conocer. Así, el alumno investiga, experimenta, a partir de su propio conocimiento". López (2011) encontró que los profesores valoran el desarrollo de habilidades personales de los alumnos a través del trabajo colaborativo con la Wiki (entre los que se encuentra Intranet) (0.728 correlación de Pearson), tanto para los estudios como para su trabajo futuro en el mundo laboral y que mediante el aprendizaje colaborativo los alumnos han participado en la construcción de conocimientos relacionados con sus estudios; entre las herramientas más utilizadas está el editor de contenidos web Joomla. Otro de los resultados encontrados en la presente investigación concuerda con González (2009), quien encontró que los participantes del estudio no cuentan con sistemas de codificación de flujos de conocimiento, pero parcialmente, esto incide en forma negativa en el desarrollo de las investigaciones, puesto que genera retrasos en su desarrollo, fuga de información y pérdida de tiempo. También se ha encontrado en esta investigación una alta correlación de 0.864 en que el Intranet facilita almacenar, recuperar y procesar la información, lo cual lo sostiene en un $90 \%$ los docentes y en un $100 \%$ los estudiantes, podemos corroborar lo que menciona Hopkins (2006) el utilizar estrategias de gestión de conocimiento permite encontrar que los profesores genere un crecimiento y mejora en sus métodos de enseńanza y de conocimiento, redundando en la mejora del proceso de aprendizaje de los estudiantes. 


\section{REFERENCIAS BIBLIOGRÁFICAS}

Abad, S. (2010). ¿Qué es una intranet y qué aporta a un Centro educativo? [En línea]. Recuperado de: http://elblogenelaula.wordpress.com/2010/01/30/¿que-es-una-intranet-y-que-aporta-a-un-centro-educativo/

Aportela, IM. (2007). Intranets: las tecnologías de información y comunicación en función de la organización. Acimed 2007; 16(4). Recuperado de: http://bvs.sld.cu/revistas/aci/ vol16_4_07/aci041007.html

Barney, J.B. (2012). Firm resource and sustained competitive advantage. N. York: Journal of Management, 17 (1) 41-56

Bueno, E. (2008). Enfoques principales en Dirección del Conocimiento (Knowledge Management) y tendencias. En R. Hernández (ed.): Gestión del Conocimiento: Desarrollos teóricos y aplicaciones. Cáceres: Ediciones La Coria, Fundación Xavier de Salas.

Bueno, E. (2009). La sociedad del conocimiento: un nuevo espacio de aprendizaje de las personas y organizaciones, en La Sociedad del Conocimiento, Monografía de la Revista Valenciana de Estudios Autonómicos, Presidencia de la Generalitat Valenciana, Valencia.

Carrillo, P.M.; Anumba, C.J. y Kamara, JM. (2010). Knowledge management for construction: key IT and contextual issues. En Gudnason G, editor. Proceedings of the international conference on construction information technology, 28-30 junio, Reykjavik, Islandia: Icelandic building research institute.

Carrillo, M.V.; Castillo, A.; Gómez, L. (2010). Las nuevas tecnologías al servicio de la comunicación empresarial. Marco teórico y resultados de la investigación empírica. Hologramática 2010; Año III (4). [En línea]. Recuperado el 22 de abril de 2014 de: http://www.cienciared.com.ar/ra/ usr/3/198/hologramatica4_pp35_54.pdf

Cascante, P y Fonseca, M. (2008). Intranet: Una ventana a la comunicación interna de las organizaciones. Revista de Derecho y Tecnologías de la Información 2008(4). [En línea]. Recuperado el 22 de abril del 2014 de: http://www. uned.ac.cr/redti/cuarta/art2.pdf

Donate, M. y Guadamillas, F. (2010). Estrategia de gestión del conocimiento y actitud innovadora en empresas de Castilla-La Mancha. Un Estudio exploratorio. Rev. Investigaciones Europeas de Dirección y Economía de la Empresa. Vol. 16 (1), pp. 31-54. [En línea]. Consultado el 30 de enero del 2014 en: http://www.aedem-virtual.com/articulos/iedee/ v16/161031.pdf

Hopkins, J. (2006). Hacia un Modelo de Gestión del Conocimiento en el Colegio Peruano Británico: Diseño General y Estrategia de implantación. (Tesis de Magíster en Educación con Mención en Gestión de la Educación). Lima: PUCP.

Henao, M. y Cardona, S. (2010). Estrategias tecnológicas de gestión del conocimiento integradas con sistemas de información empresariales. Americas Conference on Information Systems (AMCIS). AMCIS 2010 Proceedings [En línea]. Consultado el 06 enero 2014 en: http://aisel.aisnet.org/cgi/viewcontent.cgi?article=1418\&context=amcis 2010

Joo, B. (2004). Análisis y propuesta de gestión pedagógica y administrativa de las TICS, para construir espacios que generen conocimiento en el Colegio Champagnat. (Tesis de Magíster en Educación con Mención en Gestión). Lima: PUCP.

Martínez, F.; Peñalver, A. y Salamanca, J. (2009). Gestión estratégica del conocimiento. España: Santander Central Hispano.

Méndez, C. (2011). Metodología: Guía para elaborar diseños de investigación en ciencias económicas, contables y administrativas. 3ra. edición, segunda impresión. Santa Fe de Bogotá: McGraw-Hill.

Nonaka, I. y Takeuchi, H. (2008). Cómo las compañías japonesas crean la dinámica de la innovación. México: Oxford University Press.

O’Brien, J. y Marakas, G. (2008). Sistemas de información gerencial. Octava edición. México: McGraw-Hill.

Pérez, D. y Solana, P. (2009). Intranets: medición y valoración de sus beneficios en las organizaciones. El Profesional de la Información 2007; 15(5) 331-41.

Quintana, Y. (2006). Gestión por el conocimiento en la carrera de Ingeniería Industrial. Administración de operaciones. Tesis en opción al título de Master en Ciencias. Facultad de Industrial Economía. Universidad de Matanzas. Cuba.

Ruiz, R. (2006). Diseño de una intranet de apoyo a la gestión académica para los colegios de la ciudad de Piura. (Tesis para optar el Título de Ingeniero Industrial y de Sistemas). Piura: Universidad de Piura. [En línea]. Consultado el 06 setiembre del 2013 en: http://www.biblioteca.udep.edu.pe/ BibVirUDEP/tesis/pdf/1_41_6_7_275.pdf

Sabino, C. (2007). El proceso de Investigación. 4. ${ }^{\text {a }}$ edición Bogotá - Colombia: Panamericana.

Schulz, M. y Jobe, L. A. (2001). Codification and tacitness as knowledge management strategies: an empirical exploration. Journal of High Technology Management Research, $12(139-95)$

Snowden, D. (2009). Liberating Knowledge. En: Reeves J, (ed.). Londres: Liberating knowledge, Caspian Publishing

Soy, C. (2009). ¿Intranets? Decididamente sí, pero... El Profesional de la Información 2009; 15(5) 328-30

Tari, J.; García, M. (2009). Dimensiones de la gestión del conocimiento y la gestión de la calidad: Una revisión de la literatura. Investigaciones Europeas de Dirección y Economía de la Empresa, 15(3) 135-148. http://dx.doi.org/10.1016/ S1135-2523(12)60105-1

Teece, DJ. (2010). Strategies for managing knowledge assets: the role of firm structure and industrial context. Long Range Planning, 33

Wiig, K.M. (2007). Integrating Intellectual Capital and Knowledge Management. Long Range Planning, 30(3). 